\title{
A Nonlinear Continuous-Discrete Filter with Model Parameter Uncertainty and Application to Anesthesia*
}

\author{
João M. Lemos, Conceição Rocha, Teresa F. Mendonça and Maria Eduarda Silva
}

\begin{abstract}
This paper addresses the problem of joint estimation of the state and parameters for a deterministic continuous time system, with discrete time observations, in which the parameter vector is constant but its value is not known, being a random variable with a known distribution. Along time, the uncertainty in the parameter induces uncertainty in the plant state. The joint probability density function (pdf) satisfies the Liouville partial differential equation that is a limit case of the Fokker-Planck equation for vanishing diffusion. The continuous-discrete filter proposed operates as follows: Between two consecutive output sampling time instants, the pdf is propagated by solving the Liouville equation for an augmented state and is then corrected by using the last observation and Bayes law. An application to state estimation of the neuromuscular blockade of patients subject to general anesthesia, where parameter uncertainty is due to inter-patient variability, is described.
\end{abstract}

\section{INTRODUCTION}

\section{A. Motivation}

Estimating the state of a system is a fundamental problem of control engineering. In the presence of uncertainty, and from a probabilistic point of view, propagating in time the probability density function (pdf) of the state variable given the observations of the system output provides complete information about its solution. Indeed, if this pdf is known, state estimates derived from criteria such as least mean squares, maximum-likelihood or maximum a posteriori estimates can be readily computed. In the case considered here of a continuous-time system with discrete time observations the uncertainty is often represented by a white noise input signal. In this case, the a priori (i.e., given no observations)

*This work was supported by FEDER funds through COMPETEOperational Programme Factors of Competitiveness ("Programa Operacional Factores de Competitividade") and by Portuguese funds through the Center for Research and Development in Mathematics and Applications (University of Aveiro) and the Portuguese Foundation for Science and Technology ("FCT-Fundação para a Ciência e a Tecnologia"), within projects PEst-C/MAT/UI4106/2011 with COMPETE number FCOMP-01-0124-FEDER-022690, INESC-ID PEst-OE/EEI/LA0021/2013 and GALENO - Modeling and Control for personalized drug administration, PTDC/SAU-BEB/103667/2008. Conceição Rocha acknowledges the grant SFRH/BD/61781/2009 by FCT/ESF.

J. M. Lemos is with INESC-ID and also with the Instituto Superior Técnico, Technical University of Lisbon, 1000-029 Lisbon, Portugal (e-mail: jlml@inesc.pt)

C. Rocha and T. Mendonça are with the Departamento de Matemática, Faculdade de Ciências da Universidade do Porto, Rua do Campo Alegre, 4169-007 Porto, Portugal, and also with the Center for Research \& Development in Mathematics and Applications (CIDMA), Universidade de Aveiro, Portugal.(e-mail: mnrocha@fc.up.pt; tmendo@fc.up.pt)

M. E. Silva is with Faculdade de Economia da Universidade do Porto, Rua Dr. Roberto Frias, 4200-464 Porto, Portugal, and also with the Center for Research \& Development in Mathematics and Applications (CIDMA), Universidade de Aveiro, Portugal.(e-mail: mesilva@fe.up.pt) state pdf can be computed by the Fokker-Planck equation (FPE), also known as the forward Kolmogorov equation [1] Although at the expense of a heavy computational load, for controlled systems the FPE can then be used to design control laws that take into account the plant state uncertainty $[2]$.

A source of uncertainty other then input stochastic disturbances is parameter uncertainty. For instance for patients subject to anesthesia, both the pharmacokinetic and pharmacodynamic responses to a drug bolus administered to induce neuromuscular blockade present strong variations from patient to patient [3]. Even if there are no input stochastic disturbances, if the model parameters are described by a random variable, the plant state at a given time instant becomes a random variable. This type of problem is addressed here by enlarging the plant state with the parameter vector. Furthermore, in order to reduce the computational burden, a limit case of the FPE in which the diffusion term vanishes is considered. This equation is known as the Liouville equation [4] after being named by Gibbs in relation to problems in statistical mechanics [5] (page 9).

In the context of this paper, the Liouville equation propagates in time the state pdf of a deterministic system whose initial condition is a random variable with known pdf. When coupled with observations made at discrete time instants using Bayes law, a nonlinear continuous-discrete filter [1] that jointly estimates plant states and parameters is obtained.

\section{B. Literature review}

For linear systems and under gaussian noise, continuousdiscrete filters can be readily obtained from the Kalman filter expressions by discretizing system dynamics [6]. Since even linear plants with the state augmented by the parameter vector become nonlinear, one has to resort to nonlinear methods. Although the extended Kalman filter (EKF) provides an extension to the nonlinear case [7], this method may provide a poor approximation to the state pdf along time, that may be needed in order to evaluate the uncertainty bounds. In this respect, a better way is to propagate the state pdf using the FPE and then use Bayes law to filter this predicted density using the observations, a method already used in the early references [8], [1].

Although several methods are available, including finite differences, spectral methods and finite element methods, the numerical solution of parabolic partial differential equations such as the FPE is computationally heavy and requires the satisfaction of some conditions for convergence. In relation to continuous-discrete filters these issues lead to the consid- 
eration of algorithms whose convergence to the optimal filter can be proved, while alleviating the computational load [9].

Recently, the subject of continuous-discrete filtering is receiving significant attention. Research includes the use of Feynman path integrals to approximate solutions to the FPE [10], [11], [12], kernel density estimates again together with path integrals [13], and fast approximations [14]. Specific work on joint state and parameter estimation, in particular exploiting the use of the Liouville equation for systems without stochastic disturbances is not found in the literature.

\section{Paper contributions and organization}

The contributions of this paper consists of a continuousdiscrete filter for joint state and parameter estimation for deterministic systems subject to parameter uncertainty, and its application to the study of neuromuscular blockade of patients subject to general anesthesia. Both simulation and real clinical data based examples are presented.

The paper is organized as follows. After the introduction (this section), in which the work to present is motivated, the relevant literature is reviewed and the contributions are stated in concise terms, the continuous-discrete filter is described in section II, including the model, the Liouville equation used to propagate the augmented state pdf and the bayesian correction based on the observations. Section III presents a simple but non-trivial example in which the main concepts are illustrated. Section IV describes the application to state estimation in neuromuscular blockade with interpatient variability. Finally, section V draws conclusions and discusses future work.

\section{CONTINUOUS-DISCRETE FILTER}

\section{A. State model}

Consider a nonlinear system modeled by the state equation

$$
\frac{d x}{d t}=f(x, \theta)
$$

with state $x \in \mathbb{R}^{n}$, parameters $\theta \in \mathbb{R}^{n_{p}}, f: \mathbb{R}^{n} \times \mathbb{R}^{n_{p}} \rightarrow \mathbb{R}^{n}$.

The initial condition $x\left(t_{0}\right)=x_{0}$ is, in general, a random variable with pdf $p_{x}\left(x\left(t_{0}\right), t_{0}\right)$. The parameter vector is constant, being a random variable for which the only a priori information about it is the pdf $p_{\theta}(\theta)$.

Depending on the value of $\theta$, different trajectories of the state $x$ are obtained. At each time $t$, the state $x=x(t, \theta)$ is therefore also a random variable characterized by a pdf $p_{x}(x, t)$, even if $x\left(t_{0}\right)$ is deterministic.

In order to compute $p_{x}(x, t)$, consider the augmented state $z \in \mathbb{R}^{n+n_{p}}$ given by

$$
z(t)=\left[\begin{array}{c}
x(t) \\
\theta
\end{array}\right]
$$

The augmented state verifies the equation

$$
\frac{d z}{d t}=F(z)
$$

with

$$
F(z)=\left[\begin{array}{c}
f(x) \\
0
\end{array}\right]
$$

and initial conditions that derive from (1) in a straightforward way. Computing $p(z, t)$ provides not only the required information on $p_{x}(x, t)$ but also on $p_{\theta}(\theta, t)$.

\section{B. Liouville equation}

A partial differential equation for the pdf of the augmented state $p(z, t)$ can be obtained by considering a stochastic differential equation corresponding to (1), writing the associated Fokker-Planck equation and then making the input noise power to vanish, eliminating the diffusion term [1]. This results in the Liouville equation

$$
\frac{\partial}{\partial t} p(z, t)+\sum_{i=1}^{n+n_{p}} \frac{\partial}{\partial z_{i}}\left(F_{i}(z) p(z, t)\right)=0
$$

or, in a more compact way,

$$
\frac{\partial p}{\partial t}+\frac{\partial p}{\partial z} F+\operatorname{tr}\left(\frac{\partial F}{\partial z}\right) p=0
$$

where

$$
\frac{\partial p}{\partial z}=\left[\begin{array}{ccc}
\frac{\partial p}{\partial z_{1}} & \cdots & \frac{\partial p}{\partial z_{n+n_{p}}}
\end{array}\right]
$$

is the gradient of $p$ with respect to $z$ and

$$
\frac{\partial F}{\partial z}=\left[\frac{\partial F_{i}}{\partial z_{j}}\right]
$$

is the Jacobian matrix of $F$ with respect to $z$ at time $t$.

The solution of (4) (or (5)) is subject to the initial condition $p\left(z, t_{0}\right)$ specified and to the boundary conditions $\lim z \rightarrow \pm \infty=0$.

While the Fokker-Planck is a parabolic equation, the Liouville equation is a hyperbolic equation. Moreover, the Liouville equation can be solved exactly.

\section{Solution of the Liouville equation}

The following proposition is proved in the appendix:

\section{Proposition 1}

Consider the two following PDEs:

$$
\frac{\partial p_{1}}{\partial t}+\frac{\partial p_{1}}{\partial z} F=0
$$

and

$$
\frac{\partial p_{2}}{\partial t}+\operatorname{tr}\left(\frac{\partial F}{\partial z}\right) p_{2}=0
$$

with initial conditions $p_{i}\left(z, t_{0}\right), i=1,2$, given.

A) Equation (6) has the solution

$$
p_{1}(z, t)=p_{1}\left(z-\int_{t_{0}}^{t} F(z(\sigma)) d \sigma, t_{0}\right) .
$$

B) Equation (6) has the solution

$$
p_{2}(z, t)=p_{2}\left(z, t_{0}\right) \exp \left[-\int_{t_{0}}^{t} \operatorname{tr}\left(\frac{\partial F}{\partial z}\right) d \sigma\right] .
$$

C) Denote the integral operators that map the initial conditions of, respectively, (6) and (7), at time $t_{0}$ to the solution at time $t$ by $T_{t, t_{0}}^{i}, i=1,2$. These operators are defined by (8) and (9). The solution of the Liouville equation 
(5) is obtained by the composition of the two solutions of

(6) and (7). In other words, the solution of (4) is given by

$$
p(z, t)=T_{t, t_{0}}^{2} T_{t, t_{0}}^{1} p\left(z, t_{0}\right) .
$$

\section{Observation model and filtering}

Add now to (3) the observations model

$$
y(t)=h(z(t))+\eta(t)
$$

where $y \in \mathbb{R}$ is the observation, $h: \mathbb{R}^{n+n_{p}} \rightarrow \mathbb{R}$ and $\{\eta\}$ is a sequence of independent, identically distributed gaussian random variables with zero mean and variance $\sigma_{\eta}^{2}$. Assumed that the observations are made at discrete time instants $t_{0}, t_{1}, \ldots, t_{k}$ and define the set of observations

$$
Y^{t_{k}}=\left\{y\left(t_{0}\right), y\left(t_{1}\right), \ldots, y\left(t_{k}\right)\right\} .
$$

With an abuse of notation we consider pdf conditioned on sets like $Y^{t_{k}}$ where the conditioned should be on the $\sigma$ algebras associated to these sets.

The continuous-discrete filtering problem consists in estimating $z\left(t_{i}\right)$ from the observations of $y$ up to time $t$. Whatever the estimation criterion might be, the full information required to compute the estimate is contained in the pdf of $z$ given the observations. This pdf is computed according to the following steps:

\section{Continuous-discrete filter}

Let $p\left(z\left(t_{k-1}\right) \mid Y^{t_{k-1}}\right)$ (the "filtered pdf") be available from the previous steps of the algorithm.

- Prediction step: Compute $p\left(z\left(t_{k}\right) \mid Y^{t_{k-1}}\right)$ (the "predicted pdf") by propagating from time $t_{k-1}$ until $t_{k}$ the pdf $p\left(z\left(t_{k-1}\right) \mid Y^{t_{k-1}}\right)$. For this sake solve the Liouville equation (5) in the time interval that starts at $t_{k-1}$ and ends at $t_{k}$, taking as initial condition $p\left(z\left(t_{k-1}\right) \mid Y^{t_{k-1}}\right)$.

- Filtering step: Compute the filtered pdf at time $t_{k}$ using

$$
p\left(z\left(t_{k}\right) \mid Y^{t_{k}}\right)=\mathcal{K}\left(t_{k}\right) p\left(y\left(t_{k}\right) \mid z\left(t_{k}\right)\right) p\left(z\left(t_{k}\right) \mid Y^{t_{k-1}}\right),
$$

where $\mathcal{K}$ is a normalizing constant that depends on time.

The proof of (13) uses a well known argument based on Bayes law and is done in the appendix for the sake of completeness.

The pdf $p\left(y\left(t_{k}\right) \mid z\left(t_{k}\right)\right)$ depends on the observations (sensor) model. In the situation (11),

$$
p\left(y\left(t_{k}\right) \mid z\left(t_{k}\right)\right)=C_{\eta} \exp \left\{-\frac{1}{2 \sigma_{\eta}^{2}}\left[y\left(t_{k}\right)-h\left(z\left(t_{k}\right)\right)\right]^{2}\right\},
$$

with $C_{\eta}$ a normalizing constant.

\section{E. Numerical implementation}

In order to write a numerical algorithm that implements an approximation of the continuous-discrete filter described in sub-section II-D, define a uniform grid of sampling instants $t_{k}$ by

$$
t_{k+1}=t_{k}+\Delta_{t}
$$

for $k=0,1, \ldots, N_{k}$ and $\Delta_{t}$ a constant, and a non-uniform grid of state points $z_{j}\left(t_{k}\right)$ by

$$
z_{j}\left(t_{k}\right)=z_{j}\left(t_{k}-1\right)+F\left(z_{j}\left(t_{k}\right)\right) \Delta_{t}
$$

for $j=0,1, \ldots, N_{x}$. With this definition of the state grid, the grid points are always over the characteristic lines of (6), greatly simplifying the prediction.

\section{Filter numerical implementation}

Let $P(j, k)=p\left(z_{j}, t_{k}\right)$. Initialize this variable for $k=0$ and recursively perform the following steps:

1) $\hat{P}(j, k)=P(j, k-1) \exp \left\{\operatorname{tr}\left(\frac{\partial f}{\partial x}\right) \Delta_{t}\right\}$

2) $P(j, k)=$

$$
=\mathcal{K}_{1}\left(t_{k}\right) \exp \left\{-\frac{1}{2 \sigma_{\eta}^{2}}\left[y\left(t_{k}\right)-h\left(z_{j}\left(t_{k}\right)\right)\right]\right\} \hat{P}(j, k)
$$

It is remarked that, due to the special structure of $F$, with the last $n_{p}$ entries equal to zero,

$$
\operatorname{tr}\left(\frac{\partial F}{\partial z}\right)=\operatorname{tr}\left(\frac{\partial f}{\partial x}\right)
$$

thereby avoiding useless, but time consuming, multiplications by zero. Similar simplifications can be made in the Liouville equation and its solution.

\section{SYNTHETIC EXAMPLE}

Consider a nonlinear system modeled by the state equation

$$
\frac{d x}{d t}=-k \alpha x(t)
$$

with both state $x \in \mathbb{R}$ and parameter $\alpha \in \mathbb{R}$, and observations model given by

$$
y(t)=x(t)+\eta(t),
$$

with $\eta(t)$ a gaussian white noise with standard deviation $\sigma_{\eta}$.

Assume for the initial condition $x(0)=x_{0}$ a gaussian distribution with mean $\mu_{x_{0}}$ and standard deviation $\sigma_{x_{0}}$; for the parameter $\alpha$ a lognormal distribution with parameters $\left(\mu_{\alpha}, \sigma_{\alpha}\right)$ and $k$ a known scale parameter. The initial pdf for this system is given by (20)

$$
\begin{aligned}
& p(x, \theta, 0)= \\
& =\frac{1}{2 \pi \sigma_{\alpha} \sigma_{x_{0}} \theta} \exp \left\{-\frac{\left(\log \alpha-\mu_{\alpha}\right)^{2}}{2 \sigma_{\alpha}^{2}}-\frac{\left(x_{0}-\mu_{x_{0}}\right)^{2}}{2 \sigma_{x_{0}}^{2}}\right\},
\end{aligned}
$$

and the pdf time evolution resulting from the Liouville equation is given by (21)

$\hat{p}(x, \alpha, t)=p\left(x-\int_{t 0}^{t}-k \alpha x\left(t^{*}\right) d t^{*}, \alpha, t_{0}\right) \exp \left\{k \alpha\left(t-t_{0}\right)\right\}$.

Suppose $k=10, \mu_{x_{0}}=100, \sigma_{x_{0}}=10, \mu_{\alpha}=-3.3, \sigma_{\alpha}=$ $0.16, \sigma_{\eta}=5$ and a case to be filtered corresponding to $\alpha=$ 0.005 and $x_{0}=140$. For this example, the time evolution of the joint probability density based on the observed $y(t)$ is plotted in Fig. 1 for three different instants, $t=3,10$ and 
20 t.u.. From this representation it is clear that even in this case where extreme values of both variables, $x_{0}$ and $\alpha$, are used the joint pdf is converging for the true set of values.

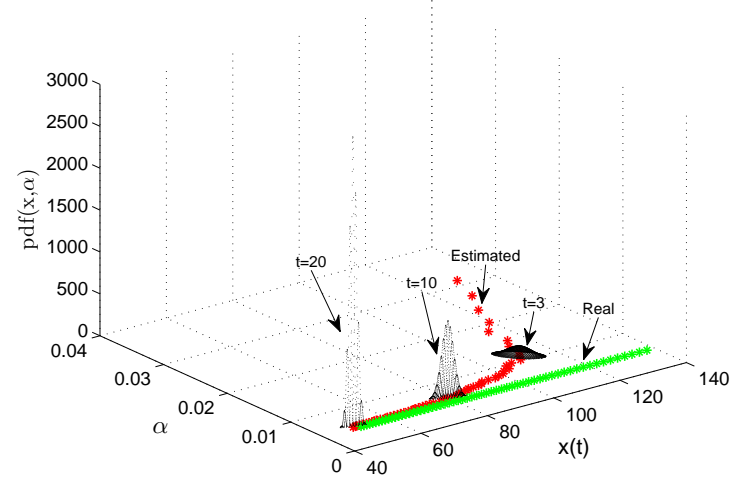

Fig. 1. Synthetic example. The a posteriori parameter and state joint density at different time instants.

The temporal evolution of the true value of the parameter $\alpha$ (that is constant) and its estimate are plotted in the plane $[\alpha, t]$ in Fig. 2, for $t \in\{3,5,7,10,13,17,29\}$ t.u..

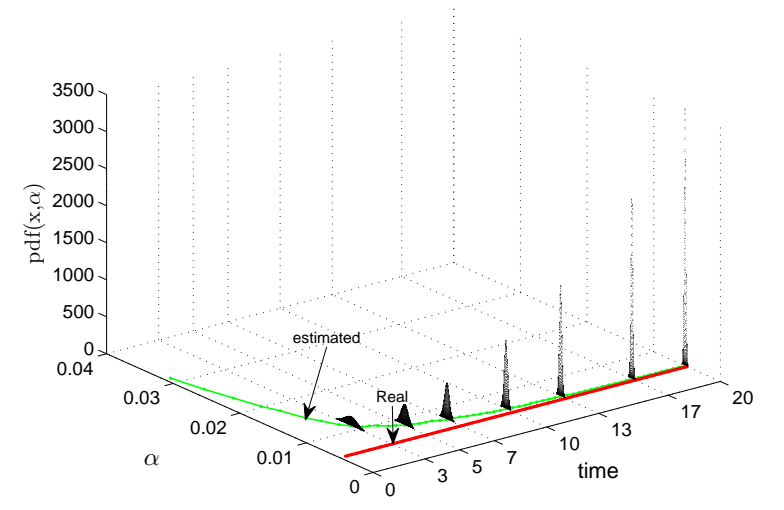

Fig. 2. Synthetic example. Projection along the parameter axis of the $a$ posteriori parameter and state joint density at different time instants.

As a complementary visualization, Fig. 3 presents the projection along the state axis of the a posteriori parameter and state joint density at different time instants.

After an initial acquisition transient, the estimates track tightly their true values. For time $t \approx 13$ t.u. both the estimated values, state and parameter, are equal to the true value of the variable.

\section{APPLICATION TO ANESTHESIA}

\section{A. Neuromuscular blockade model}

Consider now the neuromuscular blockade of patients subject to general anesthesia induced by atracurium administration. The dynamic system for the neuromuscular blockade may be modeled by (22) and (23). The linear part of the model, (22), relates the input of the system, i.e. the drug

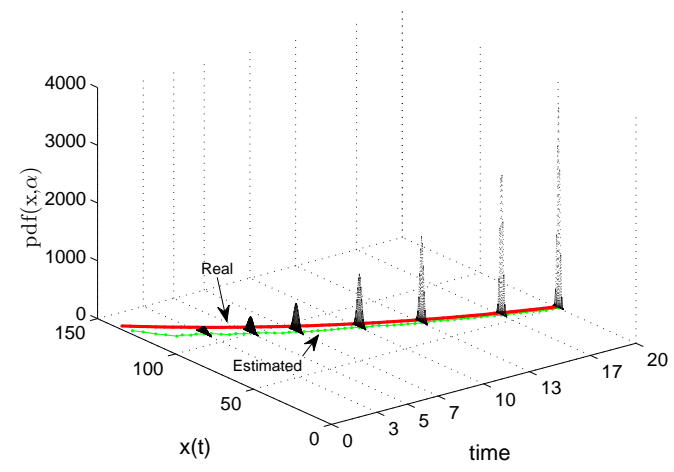

Fig. 3. Synthetic example. Projection along the state axis of the a posteriori parameter and state joint density at different time instants.

infusion rate or atracurium dosage $u(t)$ to the state variable $x_{3}(t)$.

$$
\frac{d x}{d t}=\left[\begin{array}{ccc}
-10 \alpha & 0 & 0 \\
4 \alpha & -4 \alpha & 0 \\
0 & \alpha & -\alpha
\end{array}\right] x(t)+\left[\begin{array}{c}
10 \alpha \\
0 \\
0
\end{array}\right] u(t),
$$

where $x(t)=\left[x_{1}(t), x_{2}(t), x_{3}(t)\right]$ and $\alpha$ is an unknown patient dependent parameter [17].

The observation model is given by the Hill equation

$$
y(t)=\frac{100}{1+\left(x_{3}(t) / 3.2425\right)^{\gamma}}+\eta(t),
$$

where $y$ is the observation of the blockade level $r, \gamma$ is a patient dependent parameter [17] and $\eta$ is assumed as a gaussian noise with standard deviation $\sigma_{\eta}=3$.

Due to clinical reasons, the initial conditions are $x_{1}(0)=$ $5000 \alpha$ and $x_{2}(0)=x_{3}(0)=0$. Based on [18] the following distributions are considered for $\alpha$ and $\gamma: \log (\alpha) \sim$ $\mathrm{N}\left(-3.287,0.158^{2}\right), \log (\gamma) \sim \mathrm{N}\left(0.9812,0.3458^{2}\right)$.

\section{B. Simulated neuromuscular blockade example}

To illustrate the method in the context of anesthesia, observations of the neuromuscular blockade are simulated from the above equations, (22) and (23), with $\alpha=0.01$, $\gamma=6.5$ and $u(t)$ chosen to be an initial drug bolus.

The observed signal $y(t)$ together with the simulated "true" blockade level $r(t)$ and the estimated signal are represented in the first panel of Fig. 4. The time evolution of the parameter estimates is represented in the middle, $\alpha$, and bottom, $\gamma$, panels of Fig. 4 where, for the sake of completeness a confidence region of the form $\hat{\alpha} \pm \sigma_{\hat{\alpha}}$, and $\hat{\gamma} \pm \sigma_{\hat{\gamma}}$, respectively is added. For this system the initial information conveyed by the response to the initial input bolus is not enough to lead to the convergence of $\hat{\alpha}$ and $\hat{\gamma}$. However, convergence is attained after some time. The estimates for $\alpha$ are quite stable and the estimates for $\gamma$, although presenting some oscillation, are close to the true value in the sense that the true value (in red) is almost always inside the interval $\hat{\gamma} \pm \sigma_{\hat{\gamma}}$ (in green). It is remarked that the values for the parameters $\alpha$ and $\gamma$ used in this example may be considered extreme values for their distributions. 


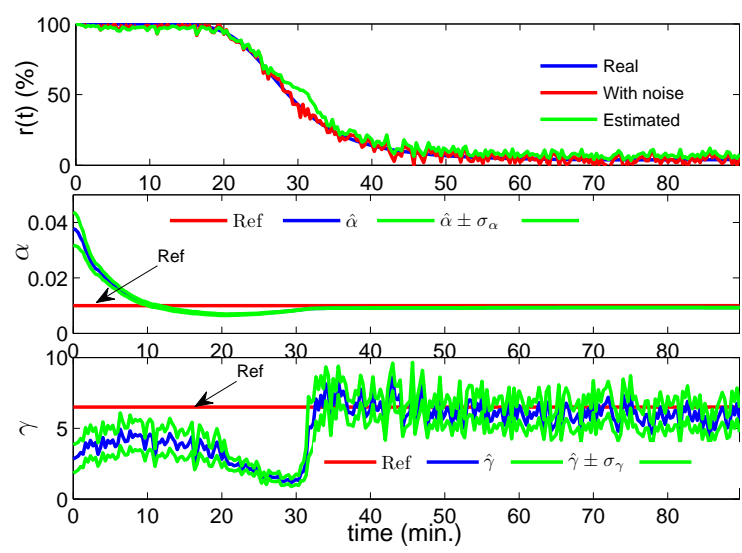

Fig. 4. Simulated neuromuscular blockade example.

\section{Results with clinical data}

When dealing with clinical data, the parameters are patient dependent and unknown and only the neuromuscular blockade records $y(t)$ are observed. Note that these records, represented in Fig. 5 for 84 patients, present large interindividual variability. Fig. 5 further represents the mean of $r(t), \mu_{r}(t)$ (in red), obtained from the solution of the Liouville equation as well as the regions $\mu_{r}(t) \pm \sigma_{r}(t)$ (in green) and $\mu_{r}(t) \pm 3 \sigma_{r}(t)$ (in yellow). From this representation it is clear that the Liouville solutions reflect the intervariability observed in these records.

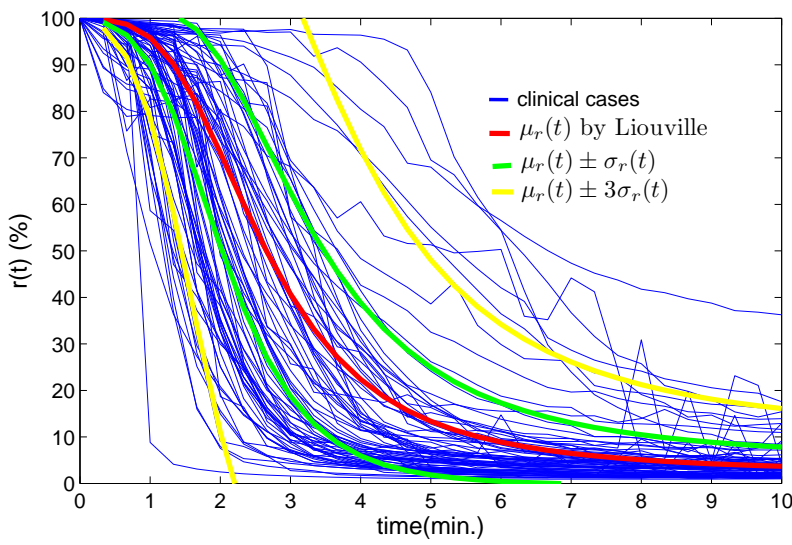

Fig. 5. 84 clinical data on blue, mean $r(t)$ give by Liouville on red and the mean added or subtracted by one (or three), standard deviation on green (on yellow).

An example of the results from the application of the continuous-discrete filter to a clinical record of the neuromuscular blockade is represented in Fig. 6. The observed signal $y(t)$ together with the filtered signal (by another technique used in these cases) and the estimated signal are represented in the first panel of Fig. 6. The time evolution of the parameter estimates is represented in the middle, $\alpha$, and bottom, $\gamma$, panels of Fig. 6 where a confidence region of the form $\hat{\alpha} \pm \sigma_{\hat{\alpha}}$, and $\hat{\gamma} \pm \sigma_{\hat{\gamma}}$, respectively is added.

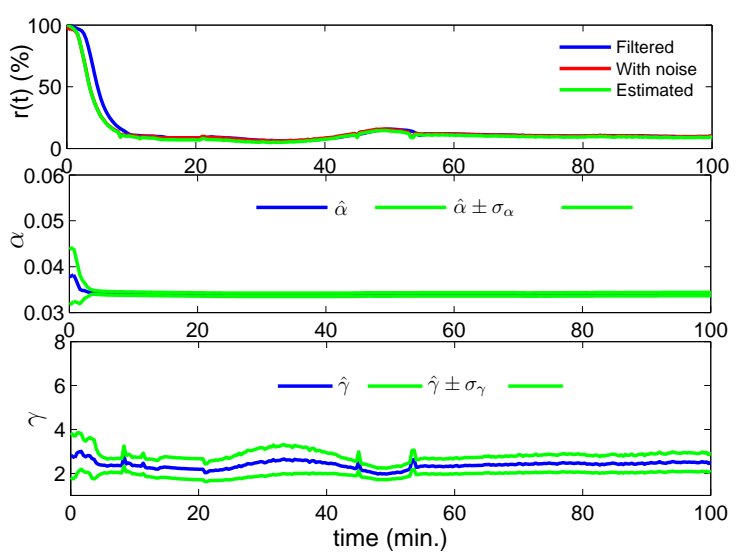

Fig. 6. Results from the application of the filter to clinical data records from neuromuscular blockade induced by atracurium.

\section{CONCLUSIONS AND FUTURE WORK}

\section{A. Conclusions}

A nonlinear continuous-discrete filter that takes into consideration a probabilistic description of uncertainty in model parameters is presented. The algorithm relies on the propagation of the a priori pdf of the state and its correction using Bayes law to obtain the filtered pdf of states and parameters given the observations in discrete time. Considering that the diffusion terms in the plant model are assumed to vanish, and that the parameters are constant, allows to propagate the pdfs using Liouville equation, instead of the Fokker-Planck equation, implying a significant reduction in the computational load. The filter was applied to study the evolution of the neuromuscular-blockade of patients subject to general anesthesia. Results related to actual clinical data are presented in order to evaluate the variability of the real data with the one predicted by the Liouville equation.

\section{B. Future Work}

A natural extension is the consideration of uncertain timevarying parameters. One possibility to model time varying parameters is to assume a model by a stochastic differential equation corresponding to (3), with the parameter equations driven by white noise (with an abuse of language), creating a so called diffusion term. In this case, the pdf of the augmented state verifies a Fokker-Planck equation, instead of a Liouville equation. Although, in general, the FPE cannot be solved in closed form, it is possible to use semigroup techniques to extend (10) in order to obtain a first order approximation, valid for $t-t_{0}$ small. This is the so called Trotter's formula [15], [16]. Other possibilities consist, of course, in the use of other methods for sampling the FPE in relation to continuous-discrete filters, such as the ones described in [12], [13], [14], and together with the augmented state.

Another continuation of the work is the development of optimal controllers, extending [2] to consider parameter uncertainty, and in applications to receding horizon optimization. 


\section{REFERENCES}

[1] A. H. Jazwinski. Stochastic processes and filtering theory. Academic Press, New York and London, 1970.

[2] K. Ohsumi and T. Ohtsuka. Nonlinear Receding Horizon Control of Probability Density Functions. Prep. 8th IFAC Symp. on Nonlinear Control Systems, NOLCOS 2010, Univ. Bologna, Italy, 2010, 735-740.

[3] J. M. Lemos, H. F. Magalhães, T. mendonça and R. Dionísio. Control of Neuromuscular Blockade in the Presence of Sensor Faults. IEEE Trans. Biomedical Eng., vol. 52, n 11, Nov. 2005, pp. 1902-1911.

[4] R. Brockett, Notes on the Control of the Liouville Equation, ch. 2 of Control of Partial Differential Equations, P. Cannarsa and J.-M. Coron (eds.). Springer, 2010.

[5] J. W. Gibbs. Elementary Principles in Statistical Mechanics, Scribner's sons, New York, 1902.

[6] F. L. Lewis, L. Xie and D. Poppa. Optimal and Robust Estimation, 2nd ed., CRC Press, 2008.

[7] J. B. Jørgensen. A critical discussion of the Continuous-Discrete Extended Kalman Filter. European Congress of Chemical Engineering - 6, Sept. 2007, Copenhagen.

[8] A. H. Jazwinski. Filtering for Nonlinear Dynamical Systems. IEEE Trans. Autom. Control vol. 11, pp. 765-766, 1966.

[9] S. V. Lototsky. Recursive nonlinear filter for a continuous discretetime model: separation of parameters and observations. IEEE Trans. Autom. Control, vol. 43, n 8, pp. 1154-1158.

[10] B. balaji and M. McDonald. Continuous-Discrete Filtering Using the Dirac-Feynman Algorithm. Proc. IEEE Radar Conf., RADAR 2008, 2008, pp. 1-6.

[11] B. balaji. Feynman path integrals and continuous-discrete filtering The additive noise case, Defense Research and Development Canada, Technical Memorandum, March 2009.

[12] B Balaji. Continuous-Discrete Path Integral Filtering. Entropy, vol. 11, pp.402-430, 2009.

[13] H. Singer. Nonlinear continuous-discrete filtering using kernel density estimates and functional densities. J. of Mathematical Sociology, vol. 27, pp. 1-28, 2003.

[14] T. Mazzoni. Fast continuous-discrete DAF-filters. J. Time Ser. Anal. vol. 33, pp.193-210, 2012.

[15] H. F. Trotter, On the product of semigroups of operators. Proc. American Mathematical Society, vol. 10, no. 4, pp. 545551, 1959.

[16] J. M. Lemos and J. M. F. Moura, Time sampling of diffusion systems using semigroup decomposition methods. MTNS 2004, 16th Int. Symp. on Mathematical Theory of Networks and Systems, Leuven, Belgium, 2004

[17] M. M. Silva, T. Wigren and T. Mendonça, Nonlinear Identification of a Minimal Neuromuscular Blockade Model in Anesthesia. IEEE Transactions on Control Systems Technology, vol. 20, no. 1, pp. 181$188,2012$.

[18] C. Rocha, T. Mendonça and M. E. Silva, Modelling Neuromuscular Blockade: a stochastic approach based on clinical data, Submitted to Mathematical and Computer Modelling of Dynamical Systems,2012.

[19] A. C. McBride, Semigroups of linear operators: An introdution. London: Longman Scientific \& Technical, 1987.

\section{APPENDIX}

\section{A1 - Proof of proposition 1}

The solution of the Liouville equation (5) is performed by Laplace's method [19]. For that sake let

$$
\psi(z, t)=z-\int_{t_{0}}^{t} F(z) d \sigma .
$$

The function $\psi(z, t)$ verifies the PDE (6). Introduce now the new set of coordinates $(\xi, \tau)$ defined by

$$
\begin{gathered}
\xi=g_{1}(z, t) \triangleq \psi(z, t), \\
\tau=g_{2}(z, t) \triangleq t .
\end{gathered}
$$

By the chain rule for derivatives,

$$
\frac{\partial p}{\partial z}=\frac{\partial p}{\partial \xi} \cdot \frac{\partial g_{1}}{\partial z}+\frac{\partial p}{\partial \tau} \cdot \frac{\partial g_{2}}{\partial z}
$$

$$
\frac{\partial p}{\partial t}=\frac{\partial p}{\partial \xi} \cdot \frac{\partial g_{1}}{\partial t}+\frac{\partial p}{\partial \tau} \cdot \frac{\partial g_{2}}{\partial t}
$$

From the definition of $g_{1}$ and $g_{2}$ given by $(25,26)$, these expressions reduce to

$$
\begin{gathered}
\frac{\partial p}{\partial z}=\frac{\partial p}{\partial \xi} \cdot \frac{\partial \psi}{\partial z} \\
\frac{\partial p}{\partial t}=\frac{\partial p}{\partial \xi} \cdot \frac{\partial \psi}{\partial t}+\frac{\partial p}{\partial \tau}
\end{gathered}
$$

Therefore, in the new coordinates, regrouping terms and observing that $\psi$ verifies (6), the Liouville equation is written in the form

$$
\frac{\partial p}{\partial \tau}=-\operatorname{tr}\left(\frac{\partial F}{\partial z}\right) p
$$

where $z$ is to be expressed in terms of $\xi$ and $\tau$ by solving (25) with respect to $z$.

Solving the equation of separable variables (31) and inverting the change of variables $(25,26)$ yields the solution of the Liouville equation

$$
p(z, t)=p\left(z-\int_{t_{0}}^{t} F(z) d \sigma, t_{0}\right) \exp \left\{-\int_{t_{0}}^{t} \frac{\partial F(z)}{\partial z} d \sigma\right\} .
$$

This expression is recognized as the composition of the solutions of (6) and (7).

\section{A2 - Proof of (13)}

Using known basic relationships from conditioned pdf, it follows that

$$
\begin{gathered}
p\left(z\left(t_{k}\right) \mid Y^{t_{k}}\right)=\frac{p\left(z\left(t_{k}\right), Y^{t_{k}}\right)}{p\left(Y^{t_{k}}\right)}= \\
=\frac{p\left(y\left(t_{k}\right) \mid z\left(t_{k}\right), Y^{t_{k-1}}\right) p\left(z\left(t_{k}\right), Y^{t_{k-1}}\right)}{p\left(Y^{t_{k}}\right)}
\end{gathered}
$$

and hence

$$
\begin{gathered}
p\left(z\left(t_{k}\right) \mid Y^{t_{k}}\right)= \\
=p\left(y\left(t_{k}\right) \mid z\left(t_{k}\right), Y^{t_{k-1}}\right) p\left(z\left(t_{k}\right) \mid Y^{t_{k-1}}\right) \frac{p\left(Y^{t_{k-1}}\right)}{p\left(Y^{t_{k}}\right)} .
\end{gathered}
$$

Since $z$ is a Markov process it follows that

$$
p\left(y\left(t_{k}\right) \mid z\left(t_{k}\right), Y^{t_{k-1}}\right)=p\left(y\left(t_{k}\right) \mid z\left(t_{k}\right)\right) .
$$

Furthermore,

$$
\frac{p\left(Y^{t_{k-1}}\right)}{p\left(Y^{t_{k}}\right)}=\frac{1}{p\left(y\left(t_{k}\right) \mid Y^{t_{k-1}}\right.}=\mathcal{K}\left(t_{k}\right)
$$

is a normalizing constant that does not depend on $z\left(t_{k}\right)$. Inserting (34) and (35) in (33) yields (13). 